\title{
Differential impact of adipokines derived from primary adipocytes of wild-type versus streptozotocin-induced diabetic rats on glucose and fatty acid metabolism in cardiomyocytes
}

\author{
Rengasamy Palanivel, Vivian Vu, Min Park, Xiangping Fang and Gary Sweeney \\ Department of Biology, York University, Toronto, Ontario, Canada M3J 1P3 \\ (Correspondence should be addressed to G Sweeney; Email: gsweeney@yorku.ca)
}

\begin{abstract}
The causal relationship between obesity and cardiovascular disease is extensively acknowledged; however, the exact mechanisms linking obesity and heart failure remain unclear. Here, we investigated the influence of adipokines derived from primary adipocytes on glucose and fatty acid uptake and metabolism in isolated primary cardiomyocytes. Either co-culture of these cell types or incubation with adipocyteconditioned medium significantly increased glucose uptake in cardiomyocytes. When streptozotocin-induced diabetic rats were used as a source of adipocytes, there was a lower ability to elicit glucose uptake in cardiomyocytes which corresponded with lower Akt and AMPK phosphorylation. The profile of glucose metabolism also differed with oxidation being favored upon co-culture with wild-type adipocytes whereas lactate
\end{abstract}

production was strongly induced by adipocytes from diabetic rats. Examination of fatty acid uptake revealed that stimulation only occurred in response to adipokines secreted by wild-type rat adipocytes. Importantly, oxidation of fatty acids by cardiomyocytes was decreased by adipokines derived from diabetic rat adipocytes. Analysis of adipokine profiles in diabetic rat adipocyte-conditioned medium demonstrated the most significant decreases in adiponectin and leptin with increased IL6 expression. Taken together, these data suggest that the profile of adipokines secreted by adipocytes from diabetic rats have a deleterious influence on cardiomyocyte metabolism which may be of relevance in the pathophysiology of heart failure.

Journal of Endocrinology (2008) 199, 389-397

\section{Introduction}

Obesity plays an influential role in dictating the morbidity and mortality associated with cardiomyopathy (Abel et al. 2008). This has prompted many studies searching for a more complete understanding of the factors and mechanisms involved in cardiac remodeling in obesity. Consequently, various adipokines have emerged as potential players in the pathophysiology of heart failure via endocrine effects which impact upon cardiac function (Bradham et al. 2002, Hopkins et al. 2007, Karmazyn et al. 2007). These include factors such as leptin, adiponectin, and TNF. It is also of interest to note that many of these factors have now been shown to be produced by the heart itself (Purdham et al. 2004, Pineiro et al. 2005, Guo et al. 2007).

One of the most significant remodeling events leading to heart failure is an alteration in cardiomyocyte metabolism (Lopaschuk et al. 2007). In the healthy heart, under aerobic conditions, the majority of energy required for contractile performance is derived from fatty acids while the remainder $(\sim 30 \%)$ is principally obtained via metabolism of glucose (Stanley et al. 2005, An \& Rodrigues 2006). Well-controlled fatty acid metabolism is also important to prevent triglyceride accumulation (McGavock et al. 2006), as this can lead to lipotoxic effects such as apoptosis or insulin resistance
(Borradaile \& Schaffer 2005). A decrease in glucose transport, glycolysis, and glucose oxidation, together with an increase in fatty acid uptake and oxidation, is typically observed in obesity and diabetes (Stanley et al. 2005, Abel et al. 2008).

Regulation of glucose and fatty acid metabolism by adipokines has been extensively demonstrated in liver and skeletal muscle (Badman \& Flier 2007). Recent studies have also shown that adipokines such as leptin (Palanivel et al. 2006) and adiponectin (Pineiro et al. 2005, Guo et al. 2007, Li et al. 2007, Palanivel et al. 2007) can mediate potent direct effects on cardiomyocyte glucose and fatty acid uptake and metabolism. Nevertheless, our knowledge of the effects of adipokines on cardiomyocyte metabolism is derived largely from studies adding recombinant proteins to quiescent cells. While this is a significant step in characterizing the effects of each adipokine, it is important to appreciate that, in physiological terms, crosstalk between effects induced by each adipokine may influence the cellular response to other adipokines. Therefore, to examine this scenario more carefully we established a co-culture system of primary rat adipocytes together with primary neonatal rat cardiomyocytes or the use of primary adipocyte-conditioned medium, which allows us to examine the effect of more physiologically relevant combinations of adipokines on cardiomyocyte glucose and fatty acid metabolism. We proposed that the adipocyte mixture 
derived from primary adipocytes would alter glucose and fatty acid metabolism in cardiomyocytes, together with associated intracellular signaling events. We also hypothesized that the profile of adipokines secreted by adipocytes derived from wildtype or diabetic rats would differ and may cause distinct metabolic and signaling effects in cardiomyocytes.

\section{Materials and Methods}

\section{Materials}

Primaria TM Easy GripTM (surface modified polystyrene, nonpyrogenic) tissue culture dishes and plates were from Becton Dickinson (Franklin Lakes, NJ, USA). DMEM/F12 medium, was obtained from Gibco (Grand Island, NY, USA). Gentamicin sulfate was obtained from Medlatech Inc. (Hsserndon, VA, USA) and penicillin/streptomycin from Wisent Inc. (St Foy, QC, Canada). 5-Aminoimidazole-4-carboxamide-1- $\beta$-D-ribofuranoside (AICAR) was purchased from Toronto Research Chemicals Inc. (Toronto, ON, Canada). $\left[{ }^{3} \mathrm{H}\right]$ palmitate, 2-deoxy-D- $\left[{ }^{3} \mathrm{H}\right]$ glucose, D- $\left[\mathrm{U}_{-}{ }^{14} \mathrm{C}\right]$ glucose, and $\left[1-{ }^{14} \mathrm{C}\right]$ palmitate were from Amersham. FATP1, FATP2, and FAT/CD36 were obtained from Santa Cruz Biotechnology (Santa Cruz, CA, USA). Primary antibodies for phosphoAMPK (Thr-172), phospho-Akt (Ser-473, Thr-308), ACC (Ser-79), and HRP-conjugated anti-rabbit secondary antibody were purchased from Cell Signaling Technology (Beverly, MA, USA). Enhanced chemiluminescence reagent was purchased from Perkin-Elmer Life Sciences (Boston, MA, USA). Streptozotocin (STZ) was purchased from Sigma. All other reagents were of the highest grade available.

\section{Isolation and culture of neonatal ventricular myocytes}

The care and use of the animals in the present study were in accordance with approved guidelines of the York University Animal Care Committee (Toronto, Canada). Primary cultures of cardiomyocytes were prepared from the ventricles of 2-to-3day-old Wistar rats by enzymatic digestion by using trypsin as described previously (Palanivel et al. 2007). Briefly, neonatal rats were put into a glass beaker containing cotton mass wetted with ethyl ether. After anesthesia and decapitation, hearts were taken out immediately and put into ice-cold calcium and bicarbonate free Hank's to HEPES (CBFHH) buffer, and then cut into pieces. Cells in suspension were collected after several rounds of digestion of heart pieces, for selective enrichment of cardiomyocytes; cells were then pre-plated into several $100 \times$ $20 \mathrm{~mm}$ culture dishes and incubated for $1 \mathrm{~h}$. The suspension containing unattached cardiomyocytes was then collected and seeded at a density of $1 \times 10^{6}$ cells $/ \mathrm{ml}$ in culture media (DMEM with $10 \%$ fetal bovine serum, $0 \cdot 1 \mathrm{mM} 5$-bromodeoxyuridine, $50 \mathrm{mg} / \mathrm{ml}$ gentamicin, $100 \mathrm{U} / \mathrm{ml}$ penicillin, and $100 \mathrm{mg} / \mathrm{ml}$ streptomycin). BrdU $(0.1 \mathrm{mmol} / \mathrm{l}$, Sigma $)$ included in the culture medium was to prevent proliferation of non-myocytes. More than $90 \%$ of the cells were myocytes, as evaluated by indirect immunofluorescence staining with an antibody to myosin heavy chain (MF20, a kind gift from DrJ C McDermott, York University, Toronto, Canada). After incubation at $37^{\circ} \mathrm{C}$ in humid air with $5 \%(\mathrm{v} / \mathrm{v}) \mathrm{CO}_{2}$ and $95 \% \mathrm{O}_{2}(\mathrm{v} / \mathrm{v})$ for $24 \mathrm{~h}$, the cardiomyocytes were then deprived of serum and incubated for another $24 \mathrm{~h}$ before treatment.

\section{Experimental induction of diabetes using $S T Z$}

Male Wistar rats were used at 6-8 weeks (250-300 g), and were either used directly for adipocyte isolation (wild-type) or diabetes was induced with STZ prior to adipocyte isolation. Diabetes was induced by i.p. injection of STZ (in $50 \mathrm{mM}$ citrate buffer, $\mathrm{pH} \mathrm{4.5)}$ at a dose of $100 \mathrm{mg} / \mathrm{kg}$ body weight. Diabetic animals exhibited slightly decreased body weight and blood glucose levels, measured with the OneTouch Ultra Meter glucometer (Lifescan, Burnaby, BC, Canada) upon removal of blood from the tail vein, which were found to increase to $31 \cdot 64 \pm 0 \cdot 642 \mathrm{mmol} / 1,6$ days following STZ injection.

\section{Isolation of adipocytes from epididymal adipose tissue}

Adipocytes were isolated as described previously (Vu et al. 2007), where epididymal adipose tissue was removed from 6- to 8 -week-old male wild-type and diabetic (6 days post-injection of STZ) Wistar rats, always between 1000 and $1200 \mathrm{~h}$ to avoid diurnal variations in adipokine profiles, and chopped with scissors into $2 \mathrm{ml}$ Krebs-Ringer-HEPES (KRH) buffer $(131.5 \mathrm{mM} \mathrm{NaCl}, 4.7 \mathrm{mM} \mathrm{KCl}, 2.5 \mathrm{mM} \mathrm{CaCl} 2,1.25 \mathrm{mM}$ $\mathrm{MgSO}_{4}, 2.5 \mathrm{mM} \mathrm{NaH} \mathrm{PO}_{4}, 10.0 \mathrm{mM}$ HEPES), supplemented with $1 \%$ BSA. Tissues were digested with collagenase type II $(1 \mathrm{mg} / \mathrm{ml})$ for $1 \mathrm{~h}$ at $37^{\circ} \mathrm{C}$ in a 250 r.p.m. shaker. After $1 \mathrm{~h}$ of digestion, infranatant was removed and adipocytes were washed with fresh KRH buffer. It is established that using this method of isolation effectively removed macrophages. The number of adipocytes was counted and diluted to $1 \times 10^{6}$ cells/ $\mathrm{ml}$ with 10\% FBS DMEM/F12 medium. Our initial experiments were performed using co-culture of primary rat adipocytes and cardiomyocytes; however, to avoid potential contaminating effects of adipocytes remaining in the wells, we also used adipocyte-conditioned media only. Similar results were observed and the latter approach was used in generating the data reported in this manuscript.

\section{Preparation of adipocyte-conditioned medium}

Adipocytes were stabilized in a $25 \mathrm{~cm}^{2}$ cell suspension flask for $3 \mathrm{~h}$ in a humidified atmosphere $\left(95 \%\right.$ air and $\left.5 \% \mathrm{CO}_{2}\right)$ at $37^{\circ} \mathrm{C}$. After stabilization, media was removed from adipocytes and changed to serum free DMEM/F12 media to prepare conditioned media. Conditioned media was collected from incubation of $1 \times 10^{6}$ adipocytes $/ \mathrm{ml}$ for $2 \mathrm{~h}$ in serum free DMEM/F12 medium and were used to treat primary cardiomyocytes for different experiments and various times indicated in the figure legends. 
Glucose and fatty acid uptake measurements in primary cardiomyocytes

Cardiomyocytes were cultured in 24-well plates and treated with adipocyte-conditioned media from control and diabetic rats for periods of $0.5,1,2$, and $3 \mathrm{~h}$ :insulin $(100 \mathrm{nM})$ for $20 \mathrm{~min}$ was used as positive control for both experiments. Consequently, glucose and/or fatty acid transport was assayed for 5 and $1 \mathrm{~min}$, respectively, at room temperature as described previously (Palanivel et al. 2007). Briefly, the incubation medium was aspirated, cells were washed with ice-cold saline, and $200 \mu \mathrm{l}$ potassium hydroxide $(\mathrm{KOH})$ $(1 \mathrm{~mol} / \mathrm{l})$ was added to each well. Aliquots of cell lysates were transferred to scintillation vials for radioactivity counting and the remainder was used for protein assay. Non-specific uptake was determined in the presence of cytochalasin B $(10 \mu \mathrm{mol} / \mathrm{l})$ and was subtracted from all the values. Results are calculated as pmol of glucose or fatty acid uptake/min $/ \mathrm{mg}$ protein.

\section{Determination of glucose and fatty acid oxidation in primary cardiomyocytes}

As described previously (Palanivel et al. 2007), glucose and fatty acid oxidation was measured by the production of ${ }^{14} \mathrm{CO}_{2}$ from $\mathrm{D}-\left[\mathrm{U}-{ }^{14} \mathrm{C}\right]$ glucose and $\left[1-{ }^{14} \mathrm{C}\right]$ palmitate respectively. Briefly, cardiomyocytes were seeded in $60 \times 15 \mathrm{~mm}$ Petri dishes and pretreated with adipocyte-conditioned medium from both normal and diabetic rats for the time indicated in the figure legend. Cells were then incubated with a medium containing $0 \cdot 15 \mu \mathrm{Ci} / \mathrm{ml} \mathrm{D}-\left[\mathrm{U}-{ }^{14} \mathrm{C}\right]$ glucose and/or $0 \cdot 15 \mu \mathrm{Ci} / \mathrm{ml}\left[1-{ }^{14} \mathrm{C}\right]$ palmitate for $2 \mathrm{~h}$. Each Petri dish was sealed with parafilm containing a piece of Whatman paper attached to the inside. The Whatman paper was wetted with $100 \mu$ l of phenylethylaminemethanol (1:1) to trap $\mathrm{CO}_{2}$ produced during the incubation period. After $2 \mathrm{~h}$ of incubation, $200 \mu \mathrm{l}$ of $\mathrm{H}_{2} \mathrm{SO}_{4}(4 \mathrm{~mol} / \mathrm{l})$ was added, followed by further incubation for $1 \mathrm{~h}$ at $37^{\circ} \mathrm{C}$. Finally, the pieces of Whatman paper were removed and transferred to scintillation vials for radioactivity counting. Insulin (100 nM) for glucose oxidation and AICAR $(2 \mathrm{mM})$ for fatty acid oxidation were used as positive controls.

\section{Measurement of glycogen synthesis}

Glycogen synthesis was measured by the incorporation of $\mathrm{D}-\left[\mathrm{U}-{ }^{14} \mathrm{C}\right]$ glucose to glycogen as described previously (Palanivel et al. 2007) with a few modifications. Briefly, cardiomyocytes were cultured in $35 \times 10$ dishes and pre-incubated with adipocyte-conditioned medium for various times $(0-3 \mathrm{~h})$ as indicated in the figure. Consequently, incubation with $0 \cdot 15 \mu \mathrm{Ci} / \mathrm{ml} \mathrm{D}-\left[\mathrm{U}-{ }^{14} \mathrm{C}\right]$ glucose for $2 \mathrm{~h}$ and insulin $(100 \mathrm{nM}$ for $2 \mathrm{~h}$ ) was used as a positive control. The cells were washed three times with cold PBS and lysed in $1 \mathrm{~mol} / \mathrm{KOH}$. To measure insulin-stimulated incorporation of glucose into glycogen, cell lysates were used for overnight glycogen precipitation with ethanol. Precipitated glycogen was then dissolved in water and transferred to scintillation vials for radioactivity counting.
Lactate production by cardiomyocytes

Lactate content was determined by the lactate oxidase method using a lactate assay kit (Sigma). Cells were pre-incubated with adipocyte-conditioned medium for the time indicated in the figure, and insulin $(100 \mathrm{nM})$ for $2 \mathrm{~h}$ was used as a positive control, after which the media was collected and used for analysis of lactate content and using adipocyteconditioned media not exposed to cardiomyocytes as control.

\section{Immunoblotting analysis of signaling proteins}

Cardiomyocytes were seeded on $35 \times 10 \mathrm{~mm}$ culture dishes and incubated acutely with adipocyte-conditioned medium from wild-type versus STZ-diabetic rat for different time point (0-10 $\mathrm{min})$ as indicated in the figure legend, and insulin for $10 \mathrm{~min}$ as a positive control for phosphorylation study. For total protein expression study, the cells were treated with adipocyte-conditioned medium for $3 \mathrm{~h}$. After appropriate treatment, cells were washed thrice with ice-cold PBS and lysates were prepared exactly as described previously (Palanivel et al. 2007). Prior to loading onto SDS-PAGE gels, the samples were diluted $1: 1(\mathrm{v} / \mathrm{v})$ with $2 \times$ Laemmli sample buffer $(62.5 \mathrm{mmol} / 1$ Tris- $\mathrm{HCl}[\mathrm{pH} 6 \cdot 8], 2 \%$ [w/v] SDS, $50 \mathrm{mmol} / 1$ dithiothreitol, $0 \cdot 01 \%$ [w/v] bromophenol blue). Equal amounts of cardiomyocyte proteins $(30 \mu \mathrm{g})$ were resolved by SDS-PAGE (8-12\%), and then transferred to polyvinylidene difluoride (PVDF) membranes (Bio-Rad). Membranes were probed with phosphorylation-specific antibodies against proteins of interest (phospho Akt (Thr308 and Ser473), phospho-AMPK (Thr172) and phospho-ACC (Ser79)) and antibodies against fatty acid transporter proteins such as FATP1, FATP2, and CD36. Appropriate HRPconjugated secondary antibodies (anti-rabbit at 1:10000 dilution,) were used in each case and detected by the enhanced chemiluminescence method. Densitometric images of data were quantitated using Scion Image software.

\section{Analysis of adipokines profiles in adipocyte-conditioned medium}

Adipocyte-conditioned medium obtained by culturing epididymal fat cells $\left(1 \times 10^{6}\right.$ cells $\left./ \mathrm{ml}\right)$ isolated from wild-type and STZ-diabetic rats were analyzed for adiponectin, leptin, and visfatin content by using ELISA kit (ALPCO Diagnostic, Windham, NH, USA). For quantitative analysis of free fatty acids in the conditioned medium, we used the HR series NEFA-HR(2) kit (Wako Pure Chemical Industries Ltd, Osaka, Japan). We used antibodies for resistin (AdipoGen, Seoul, South Korea), TNF, and IL6 (Cedarelane Laboratories, Burlington, Canada) to quantitatively determine changes in expression of these adipokines by immunoblotting, as described previously with slight modifications. Briefly, adipocyte-conditioned medium were diluted $1: 1(\mathrm{v} / \mathrm{v})$ with $2 \times$ Laemmli sample buffer $(62.5 \mathrm{mmol} / 1$ Tris- $\mathrm{HCl}[\mathrm{pH} 6 \cdot 8], 2 \%$ [w/v] SDS, $50 \mathrm{mmol} / 1$ dithiothreitol, $0 \cdot 01 \%$ [w/v] bromophenol blue). Equal volumes $(50 \mu \mathrm{l})$ of adipocyte-conditioned medium from 
wild-type versus STZ-diabetic rats were resolved by SDS-PAGE (12-15\%), and then transferred to PVDF membranes. Membranes were probed with specific antibodies against resistin and TNF at 1:1000 dilution and IL6 (1:500) and appropriate HRP conjugated secondary antibodies (anti-rabbit or anti-goat at 1:10 000 dilution) were used in each case and detected by the enhanced chemiluminescence method.

\section{Statistical analysis}

Data are expressed as means \pm s.E.M. Statistical analysis was undertaken using one-way ANOVA or the paired Student's $t$-test where appropriate. Differences between the groups were considered statistically significant when $P<0 \cdot 05$.

\section{Results}

We first determined the effect of primary adipocyte-conditioned medium from wild-type or STZ-diabetic rats on glucose uptake in primary neonatal cardiomyocytes. We found that incubation with adipocyte-conditioned medium from wild-type rats for times from $30 \mathrm{~min}$ to $3 \mathrm{~h}$ significantly stimulated 2-deoxyglucose transport into cardiomyocytes (Fig. 1A). Similar

A
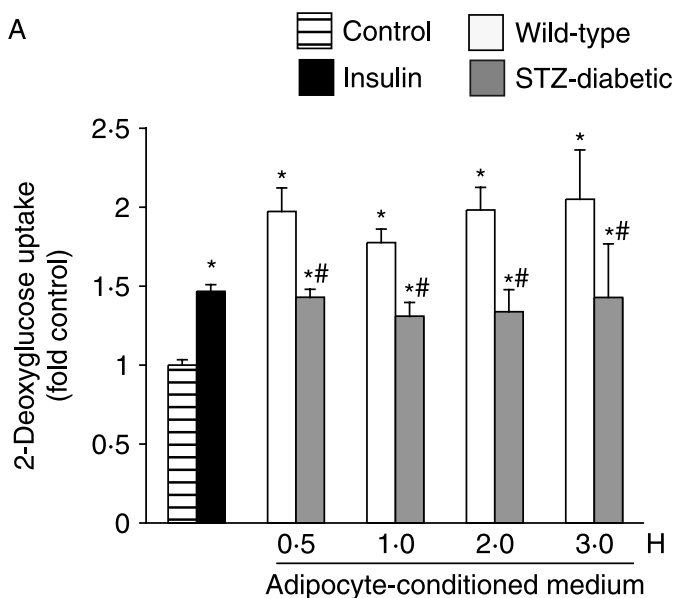

B
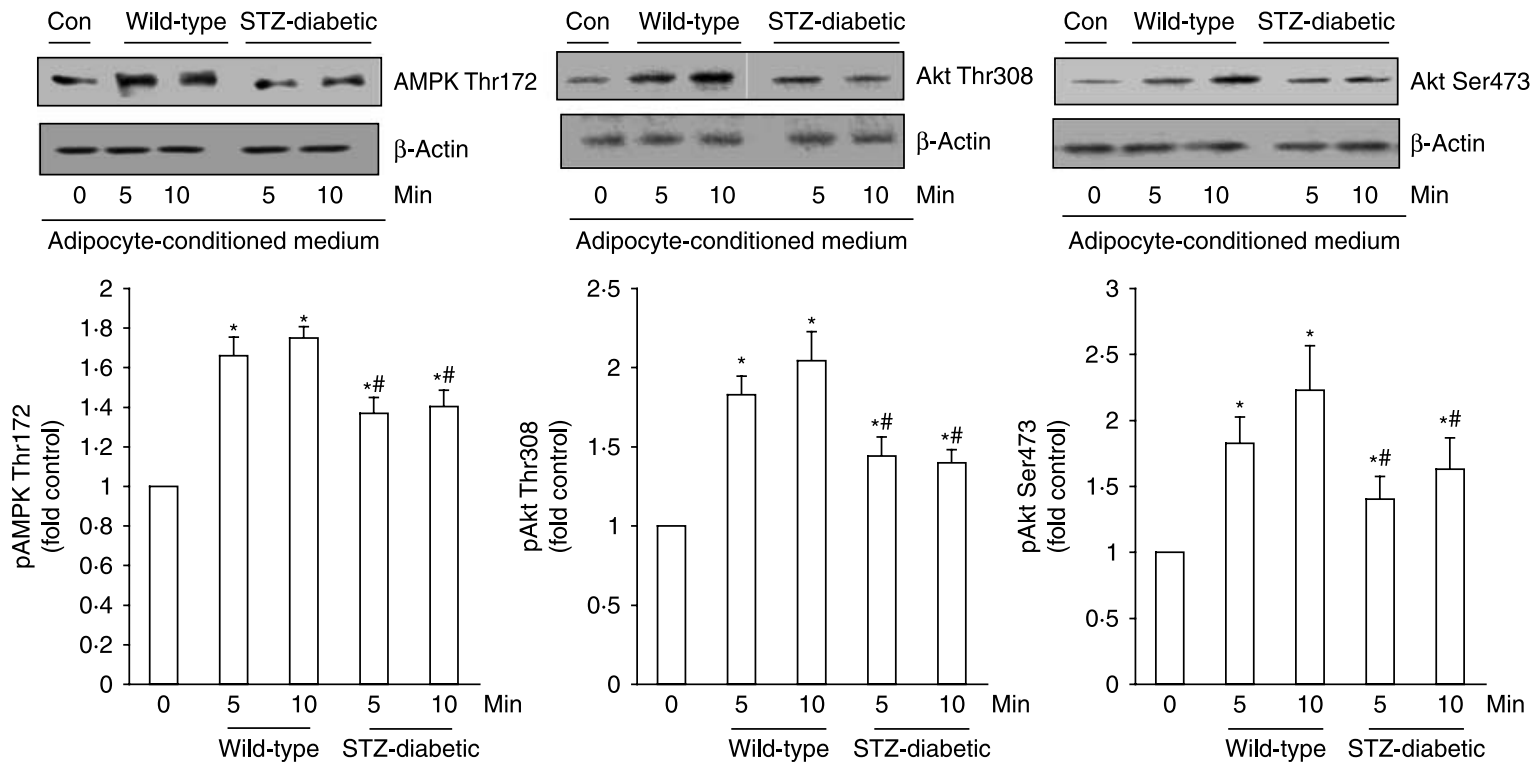

Figure 1 Regulation of glucose uptake and phosphorylation of intracellular signaling proteins by adipocyte-conditioned medium. (A) Uptake of 2-deoxyglucose was measured in response to adipocyte-conditioned medium derived from wild-type or diabetic rats ( 0 min-3 h). Insulin $(100 \mathrm{~nm})$ for $20 \mathrm{~min}$ served as a positive control. Values are expressed as mean \pm s.E.M. of $n=6 ; *$ indicates $P<0 \cdot 05$ compared with control (in the absence of adipocyte-conditioned medium) and \# indicates $P<0.05$ compared with response observed using wild-type adipocytes. (B) Co-culture effect of adipocyte-conditioned medium (5 and 10 min) on phosphorylation of AMPK (Thr172) and Akt (Thr308 or Ser473). Representative immunoblots together with quantitative analyses (mean \pm s.E.M.; $n=4$ ) are shown in all cases. 
data were obtained upon direct co-culture of primary adipocytes with cardiomyocytes (data not shown). When cardiomyocytes were treated with adipocyte-conditioned medium produced using adipocytes derived from streptozoticin-induced diabetic rats, there was a moderate stimulation of glucose uptake when compared with the control (Fig. 1A). However, the magnitude
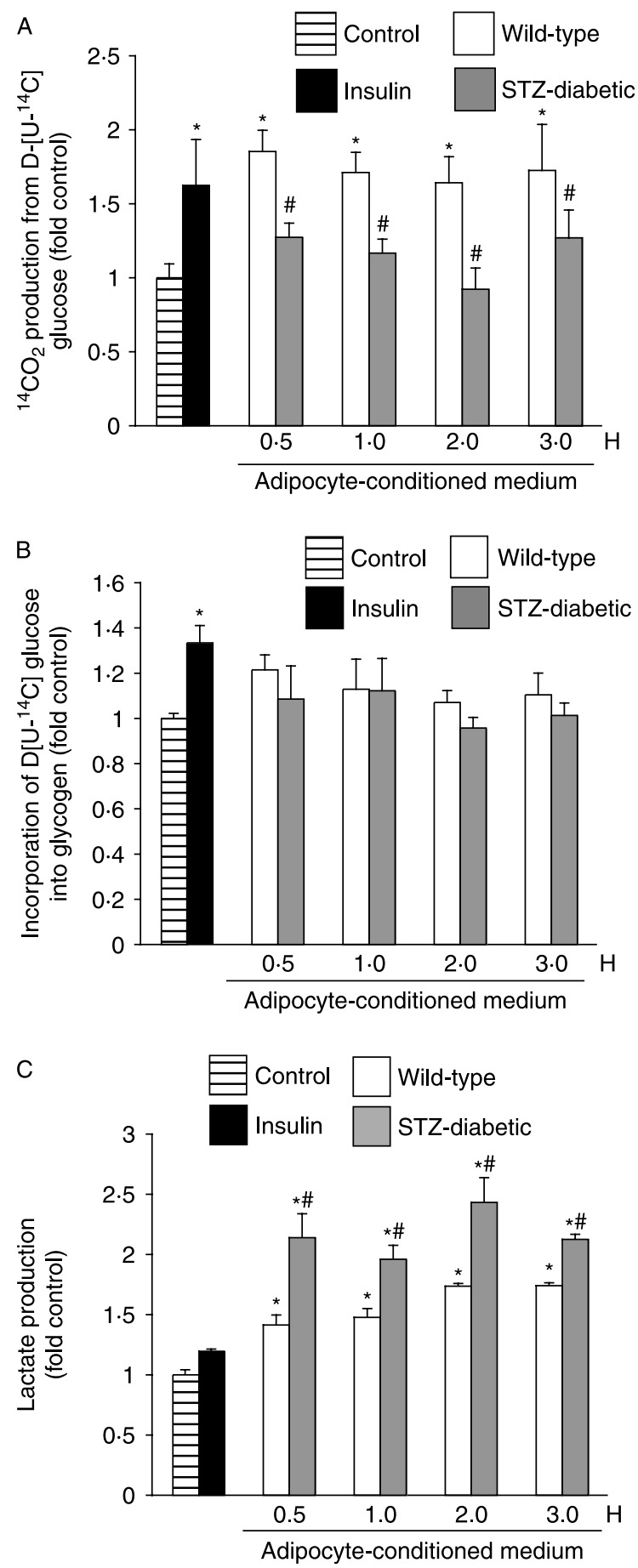

of this increase in glucose uptake was much lower than that observed in response to the adipokine mixture produced by wild-type rat adipocytes. This observation correlated with the fact that phosphorylation of both AMPK (Thr172) and Akt (Thr308 and Ser473) were stimulated to a greater degree by wild-type adipocyte-conditioned medium (Fig. 1B).

We then examined various potential routes of glucose metabolism within the cardiomyocyte. Using adipocytes derived from wild-type rats we observed that conditioned media, at times up to $3 \mathrm{~h}$, significantly elevated glucose oxidation (Fig. 2A), did not alter glycogen synthesis (Fig. 2B), and caused a slight increase in lactate production by cardiomyocytes (Fig. 2C). By contrast, there was a striking increase in lactate production when using adipocytes derived from diabetic rats (Fig. 2C) and no significant change in glucose oxidation (Fig. 2A) or glycogen synthesis (Fig. 2B).

We also observed a significant difference in the ability of wildtype and diabetic rat adipocyte-conditioned media to elicit fatty acid uptake in cardiomyocytes. At $30 \mathrm{~min}$ to $3 \mathrm{~h}$, the former significantly stimulated fatty acid uptake (Fig. 3A); however, this response was not seen in response to media prepared using adipocytes derived from diabetic rats (Fig. 3A). After $3 \mathrm{~h}$ of treatment we did not detect any change in the total expression levels of CD36, FATP1, and FATP2 (Fig. 3B).

Subsequent examination of palmitate oxidation in cardiomyocytes suggested that wild-type conditioned media (up to $3 \mathrm{~h}$ treatment) did not dramatically alter oxidation from control, although a small increase was detected at $2 \mathrm{~h}$ (Fig. 4A) and an apparent increase in ACC phosphorylation was also detected (Fig. 4B). AICAR was used as positive control in palmitate oxidation assay (Fig. 4). Interestingly, when we examined palmitate oxidation in cardiomyocytes after treatment with diabetic rat adipocyte-conditioned media, we found that the level of oxidation was even lower that basal at 1 and $2 \mathrm{~h}$ (Fig. 4).

Since we have observed differential impact of adipocyteconditioned medium obtained from wild-type and STZdiabetic rats on cardiomyocyte glucose and fatty acid uptake and metabolism, we determined changes in the secretion level of adipokines. This analysis (summarized in Fig. 5 as fold changes from values found in wild-type adipocyte-conditioned media) demonstrated that total adiponectin levels decreased most obviously $(0.46 \pm 0.10$ vs $0.084 \pm 0.008 \mu \mathrm{g} / \mathrm{ml}$ in wild-type and diabetic samples respectively). Leptin and visfatin levels also decreased significantly $(220 \cdot 34 \pm 29 \cdot 6$ vs $77 \cdot 82 \pm 13 \cdot 09 \mathrm{pg} / \mathrm{ml}$; $2.799 \pm 0.1525$ vs $1.940 \pm 0.057 \mathrm{ng} / \mathrm{ml}$ respectively) whereas

Figure 2 Regulation by adipocyte-conditioned medium on glucose oxidation, glycogen synthesis, and lactate production in primary cardiomyocytes. (A) The co-culture effect of adipocyte-conditioned medium from wild-type and/or diabetic rats (30 min-3 h) on basal ${ }^{14} \mathrm{CO}_{2}$ production from $\mathrm{D}-\left[\mathrm{U}-{ }^{14} \mathrm{C}\right]$ glucose; (B) the incorporation of $\mathrm{D}-\left[\mathrm{U}-{ }^{14} \mathrm{C}\right]$ glucose into glycogen by adipocyte-conditioned medium. (C) Lactate production by the cells. Values shown are expressed as mean \pm S.E.M. of $n=6$ experiments; ${ }^{*}$ indicates $P<0.05$ with respect to control (in the absence of conditioned medium) and \# indicates $P<0.05$ compared with response observed using wildtype adipocytes. 


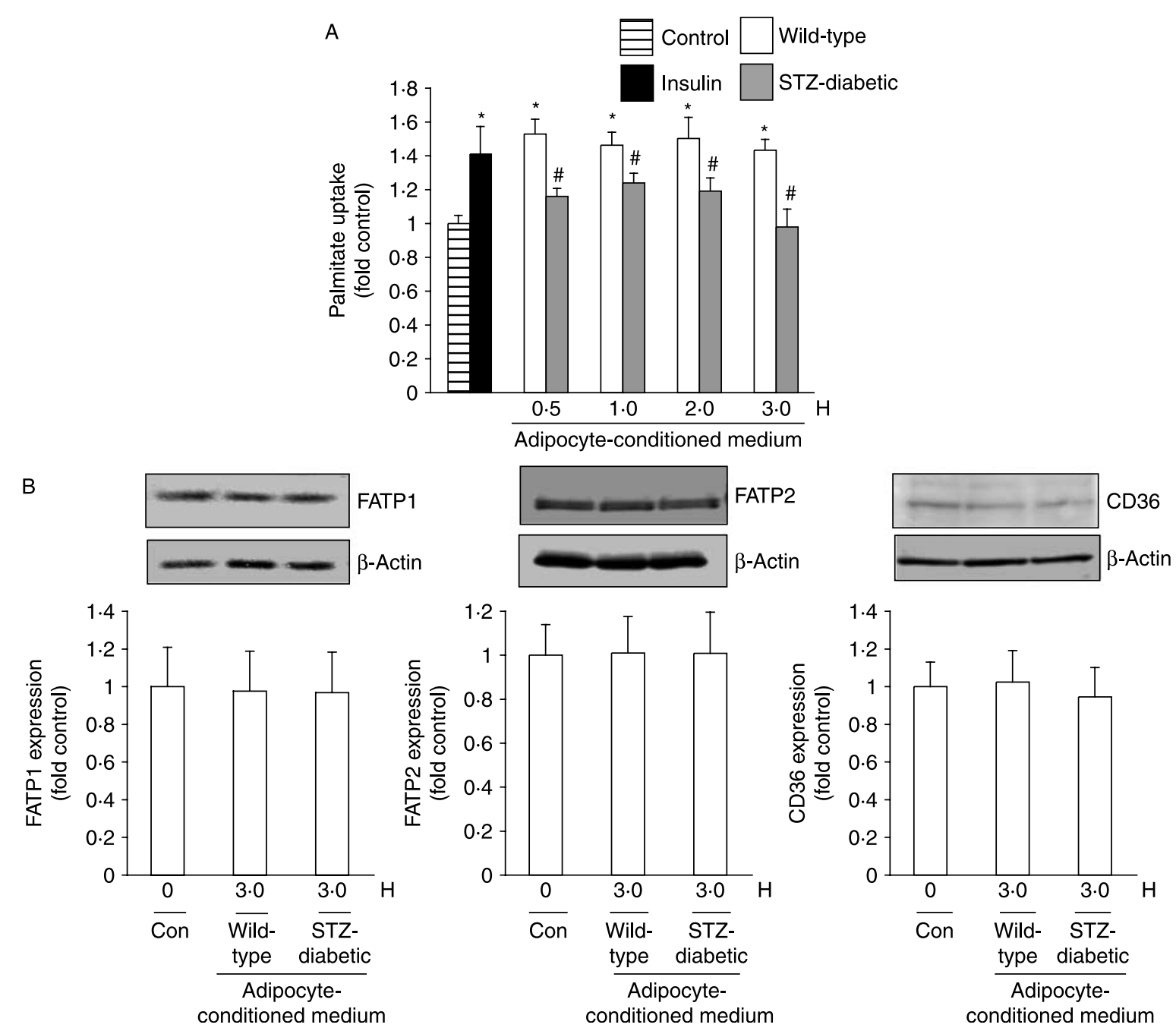

Figure 3 Impact of adipocyte-conditioned medium on fatty acid uptake and fatty acid transporter protein expression. (A) The uptake of $\left[^{3} \mathrm{H}\right]$ palmitic acid by cardiomyocytes was examined in response to adipocyte-conditioned medium from wild-type and/or diabetic rats (0-3 h). Values are expressed as mean \pm s.E.M. of $n=6 ;{ }^{*}$ indicates $P<0.05$ with respect to control (in the absence of adipocyte-conditioned medium) and \# indicates $P<0.05$ compared with response observed using wild-type adipocytes. (B) Examination of FATP1, FATP2, and CD36 protein expression in cells treated $(3 \mathrm{~h})$ with adipocyte-conditioned medium from wild-type versus diabetic rats. Representative images of four individual experiments are shown.

there was a modest and non-significant increase in resistin and TNF expression. IL6 levels increased by $34 \%$ and there was no significant difference in free fatty acid levels $(0 \cdot 0245 \pm 0 \cdot 0049 \mathrm{vs}$ $0 \cdot 0220 \pm 0 \cdot 0072 \mathrm{mM}$ respectively).

\section{Discussion}

Changes in substrate metabolism is one of the earliest measurable abnormalities in the hearts of both diabetic and obese animals and humans (Abel et al. 2008). This precedes measurable changes in in vivo cardiac function, suggesting an important causative role. Changes in myocardial substrate utilization in diabetes are well established (Stanley et al. 2005,
An \& Rodrigues 2006), and have more recently been observed in ob/ob or $\mathrm{db} / \mathrm{db}$ mouse and Zucker rat hearts prior to the onset of hyperglycemia and as early as 10 days after the initiation of high-fat feeding (Buchanan et al. 2005, Golfman et al. 2005, Park et al. 2005, Wang et al. 2005). These changes were consistently associated with increased myocardial oxygen consumption and decreased cardiac efficiency. Severely obese humans also display increased rates of fatty acid oxidation, increased myocardial oxygen consumption, and reduced cardiac efficiency (Peterson et al. 2004). Therefore, obesity and diabetes clearly precipitate heart failure at least partially via alterations in myocardial substrate metabolism.

The last decade has seen intense research interest in the role of adipokines as regulators of metabolic homeostasis, with the 


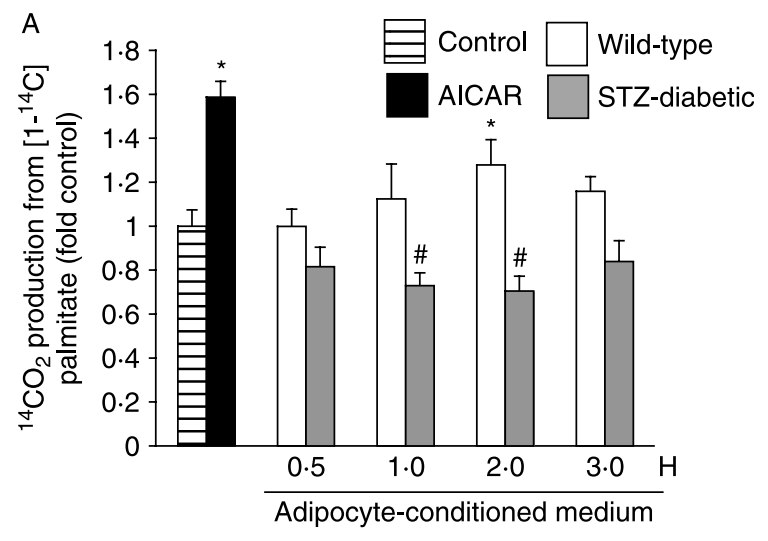

B

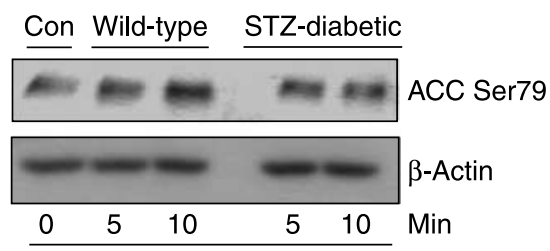

Adipocyte-conditioned medium

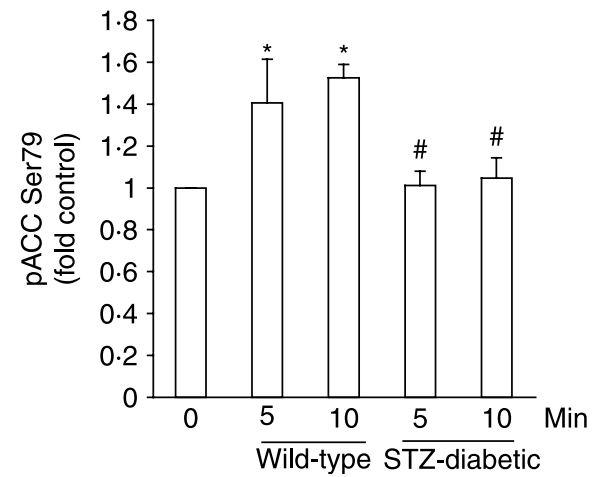

Figure 4 Regulation by adipocyte-conditioned medium on fatty acid oxidation and phosphorylation of acetyl CoA carboxylase in primary cardiomyocytes. (A) The impact of adipocyte-conditioned medium from wild-type versus diabetic rats $(0-3 \mathrm{~h})$ on ${ }^{14} \mathrm{CO}_{2}$ production from $\left[1-{ }^{14} \mathrm{C}\right]$ palmitate in cardiomyocytes. AICAR $(2 \mathrm{mM}, 2 \mathrm{~h})$ was used as a positive control. Data are representative of six independent experiments expressed as mean \pm s.E.M.; * indicates $P<0.05$ compared with control (no adipocyte-conditioned medium) and \# indicates $P<0.05$ compared with response observed using wild-type adipocytes. We examined ACC (Ser79) phosphorylation in lysates from cells treated with adipocyte-conditioned medium (0-30 min). (B) A representative image together with quantitative analysis (mean \pm S.E.M.) of four individual experiments.

majority of studies focusing on liver and skeletal muscle (Badman \& Flier 2007). Given the recent appreciation of the important contribution of adipokines to the pathophysiology of heart failure (Bradham et al. 2002, Hopkins et al. 2007, Karmazyn et al. 2007), we designed this study to examine whether adipokines directly regulate cardiomyocyte glucose and fatty acid metabolism. Reports from our laboratory (Palanivel et al. 2006, 2007) and several others (Graveleau et al. 2005, Pineiro et al. 2005, Guo et al. 2007, Li et al. 2007) have

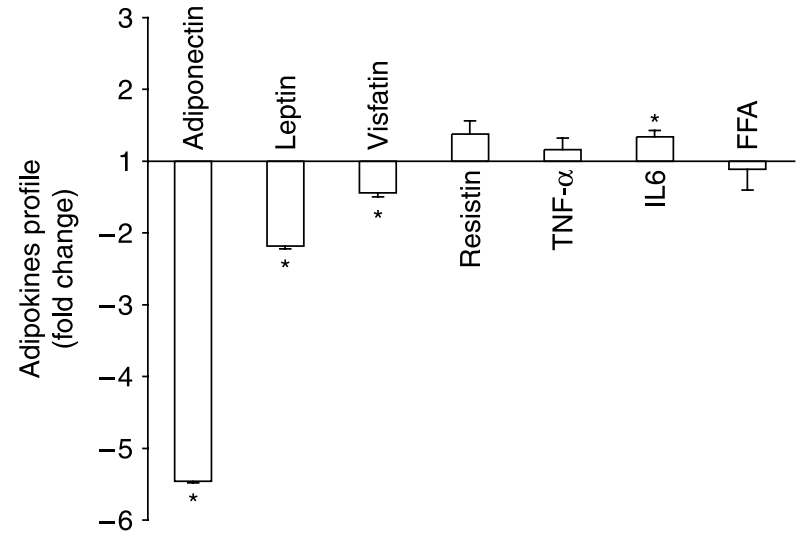

Figure 5 Changes in the adipokine profile level in wild-type versus STZ-diabetic rat adipocyte-conditioned medium. The level of adiponectin, leptin, visfatin, resistin, TNF, IL6, and free fatty acid (FFA) in adipocyte-conditioned medium is summarized here as fold changes (increase or decrease) in medium prepared using adipocytes from rats 6 days after injection of STZ compared with values found in wild-type adipocyte-conditioned media. Absolute values for the concentration of these adipokines are given where appropriate in the results section. Values shown are expressed as mean \pm S.E.M. of $n \geq 3$ experiments; * indicates $P<0 \cdot 05$ when levels in wild-type or STZdiabetic rat adipocyte-conditioned media were compared.

demonstrated direct metabolic effects of adipokines on cardiomyocyte metabolism via treating cells with individual recombinant forms of each adipokine. However, this approach inherently neglects the extensive crosstalk between signaling pathways regulated by various adipokines which exists in vivo. At the other extreme, in vivo studies are often confounded by the existence of additional inputs (e.g., neuronal). Hence, we recently established the use of a co-culture system, which allowed direct analysis of effects induced by more physiologically relevant combinations of adipokines acting in concert with one another ( $\mathrm{Vu}$ et al. 2007). In this study, we utilized a similar approach but with primary neonatal rat cardiomyocytes as our target cell.

Our results demonstrated that the profile of adipokines secreted by primary rat adipocytes stimulated glucose uptake in cardiomyocytes. This correlated with increases in both AMPK (insulin-independent) and Akt (insulin-like) signaling. Obesity and diabetes are known to be associated with decreased circulating adiponectin levels, in particular high molecular weight (HMW), which correlate closely with various aspects of cardiac remodeling and the metabolic syndrome (Liu et al. 2007, Abel et al. 2008). In this study, we used rats treated acutely with STZ to induce diabetes as an appropriate model providing a source of primary adipocytes which are known to secrete a reduced HMW adiponectin profile, indeed one which can dictate changes in glucose metabolism in skeletal muscle (Vu et al. 2007). When we used adipocytes derived from these animals as a source of adipokines, the change in glucose transport was significant but lower in magnitude than that elicited by wild-type adipokines. This is reminiscent of our previous studies in skeletal muscle cells and suggests that 
adiponectin, whose level is decreased in conditioned medium prepared using adipocytes from 3-day STZ-treated rats ( Vu et al. 2007) and even further in 6-day STZ-treated rats as shown here, may be the adipokine most likely to mediate these changes. Our studies using both recombinant adiponectin and the globular C-terminal domain of adiponectin (Palanivel et al. 2007) and those of others (Pineiro et al. 2005, Guo et al. 2007) demonstrate that this adipokine can stimulate glucose uptake in primary neonatal cardiomyocytes. These data suggest that reduced adiponectin action via reduced secretion from adipose tissue or a decrease in expression of its receptors (Guo et al. 2007) may be detrimental in terms of myocardial metabolism in obesity and diabetes. It is also feasible that many other adipokine levels change in the two types of conditioned medium used in this study and our analysis of adipokine profiles have highlighted that the significant decrease in leptin and increase in IL6 may play an important role (Ceddia et al. 2002, Carey \& Febbraio 2004).

One of the most striking observations in our study was the enhanced stimulation of lactate production induced by adipokines derived from diabetic rat adipocytes. This appeared to result as a switch of metabolism toward lactate production at the expense of glucose oxidation. The heart uses lactate as an energy source and can also produce lactate (An \& Rodrigues 2006) with the physiological consequences of increased lactate production, and decreased ATP production from glucose oxidation, being detrimental to performance of the heart (Stanley et al. 2005). Indeed, metabolic abnormalities in STZinduced diabetic hearts have been well characterized (Ramanadham et al. 1990), rendering these animals more susceptible to heart failure associated with exaggerated left ventricular remodeling including increased interstitial fibrosis and myocyte apoptosis (Shiomi et al. 2003). Specifically, cardiac utilization of lactate is reduced to an even greater extent than glucose oxidation (Chatham et al. 1999a,b). Our previous study using recombinant adiponectin showed no change in lactate production upon acute treatment times as used in this study (Palanivel et al. 2007). Hence, the loss of a permissive contribution of adiponectin in the context of adipokine mixtures or increased levels of another adipokine which directly stimulates lactate production are potential mechanisms.

As noted above, efficient uptake and oxidation of fatty acids are essential in maintaining the bulk of myocardial ATP production; yet, perturbations in fatty acid metabolism to create a discrepancy between fatty acid uptake and oxidation, as often observed in obese and diabetic individuals or animal models, can have additional consequences. For example, accumulation of lipotoxic lipid products can mediate many detrimental effects on the heart, including apoptosis, impairing contractile function, inducing or exacerbating arrhythmias and changes in cell signaling and membrane function (Schaffer 2003). Our current studies demonstrate that the ability of cardiomyocytes to uptake palmitate is enhanced by a normal adipokine mixture, which also elicits a small but significant increase in oxidation. Palmitate uptake and, in particular, oxidation are compromised by adipokines derived from diabetic rat adipocytes. Thus, although wild-type adipokine mixtures promote a beneficial increase in glucose uptake and oxidation, the increased fatty acid oxidation which we observe here may be detrimental to the heart. While our study model allows analysis of the direct influence of adipokine mixtures to be determined without potentially confounding effects of other circulating hormones or metabolites, as well as centrally mediated neuronal inputs, it is clearly important not to extrapolate our findings too far in terms of physiological significance, since the latter clearly have a significant physiological role.

In summary, these results characterize the direct effects of physiologically relevant adipokine mixtures on cardiomyocyte metabolism and highlight the differential effects of adipokine profiles derived from wild-type and STZ-induced diabetic rat adipocytes. They suggest that having an adequate amount of adipose-derived adipokines is beneficial, yet too much as is the case in obesity, too little as observed in lipoatrophy or a disturbed profile of secreted adipokines, may be detrimental to myocardial metabolism and ultimately cardiac function.

\section{Declaration of interest}

The authors declare that there is no conflict of interest that could be perceived as prejudicing the impartiality of the research reported.

\section{Funding}

Funding for this work was provided by Heart and Stroke Foundation of Canada. R P and X F also acknowledge Postdoctoral Fellowship and Graduate Studentship support, respectively, from Heart and Stroke Foundation of Canada, and G S acknowledges support from The Canadian Institutes of Health Research (CIHR) via a New Investigator award. V V is supported by a Graduate Studentship from the Canadian Diabetes Association.

\section{References}

Abel ED, Litwin SE \& Sweeney G 2008 Cardiac remodeling in obesity. Physiological Reviews 88 389-419.

An D \& Rodrigues B 2006 Role of changes in cardiac metabolism in development of diabetic cardiomyopathy. American Journal of Physiology. Heart and Circulatory Physiology 291 H1489-H1506.

Badman MK \& Flier JS 2007 The adipocyte as an active participant in energy balance and metabolism. Gastroenterology 132 2103-2115.

Borradaile NM \& Schaffer JE 2005 Lipotoxicity in the heart. Current Hypertension Reports 7 412-417.

Bradham WS, Bozkurt B, Gunasinghe H, Mann D \& Spinale FG 2002 Tumor necrosis factor-alpha and myocardial remodeling in progression of heart failure: a current perspective. Cardiovascular Research 53 822-830.

Buchanan J, Mazumder PK, Hu P, Chakrabarti G, Roberts MW, Yun UJ, Cooksey RC, Litwin SE \& Abel ED 2005 Reduced cardiac efficiency and altered substrate metabolism precedes the onset of hyperglycemia and contractile dysfunction in two mouse models of insulin resistance and obesity. Endocrinology 146 5341-5349.

Carey AL \& Febbraio MA 2004 Interleukin-6 and insulin sensitivity: friend or foe? Diabetologia 47 1135-1142.

Ceddia RB, Koistinen HA, Zierath JR \& Sweeney G 2002 Analysis of paradoxical observations on the association between leptin and insulin resistance. FASEB Journal 16 1163-1176. 
Chatham JC, Gao ZP, Bonen A \& Forder JR 1999a Preferential inhibition of lactate oxidation relative to glucose oxidation in the rat heart following diabetes. Cardiovascular Research 43 96-106.

Chatham JC, Gao ZP \& Forder JR $1999 b$ Impact of 1 wk of diabetes on the regulation of myocardial carbohydrate and fatty acid oxidation. American Journal of Physiology 277 E342-E351.

Golfman LS, Wilson CR, Sharma S, Burgmaier M, Young ME, Guthrie PH, Van Arsdall M, Adrogue JV, Brown KK \& Taegtmeyer H 2005 Activation of PPARgamma enhances myocardial glucose oxidation and improves contractile function in isolated working hearts of ZDF rats. American Journal of Physiology. Endocrinology and Metabolism 289 E328-E336.

Graveleau C, Zaha VG, Mohajer A, Banerjee RR, Dudley-Rucker N, Steppan CM, Rajala MW, Scherer PE, Ahima RS, Lazar MA et al. 2005 Mouse and human resistins impair glucose transport in primary mouse cardiomyocytes, and oligomerization is required for this biological action. Journal of Biological Chemistry $28031679-31685$.

Guo Z, Xia Z, Yuen VG \& McNeill JH 2007 Cardiac expression of adiponectin and its receptors in streptozotocin-induced diabetic rats. Metabolism 56 1363-1371.

Hopkins TA, Ouchi N, Shibata R \& Walsh K 2007 Adiponectin actions in the cardiovascular system. Cardiovascular Research 74 11-18.

Karmazyn M, Purdham DM, Rajapurohitam V \& Zeidan A 2007 Leptin as a cardiac hypertrophic factor: a potential target for therapeutics. Trends in Cardiovascular Medicine 17 206-211.

Li L, Wu L, Wang C, Liu L \& Zhao Y 2007 Adiponectin modulates carnitine palmitoyltransferase-1 through AMPK signaling cascade in rat cardiomyocytes. Regulatory Peptides 139 72-79.

Liu Y, Retnakaran R, Hanley A, Tungtrongchitr R, Shaw C \& Sweeney G 2007 Total and high molecular weight but not trimeric or hexameric forms of adiponectin correlate with markers of the metabolic syndrome and liver injury in Thai subjects. Journal of Clinical Endocrinology and Metabolism 92 4313-4318.

Lopaschuk GD, Folmes CD \& Stanley WC 2007 Cardiac energy metabolism in obesity. Circulation Research 101 335-347.

McGavock JM, Victor RG, Unger RH \& Szczepaniak LS 2006 Adiposity of the heart, revisited. Annals of Internal Medicine 144 517-524.

Palanivel R, Eguchi M, Shuralyova I, Coe I \& Sweeney G 2006 Distinct effects of short- and long-term leptin treatment on glucose and fatty acid uptake and metabolism in HL-1 cardiomyocytes. Metabolism 55 1067-1075.

Palanivel R, Fang X, Park M, Eguchi M, Pallan S, De Girolamo S, Liu Y, Wang Y, Xu A \& Sweeney G 2007 Globular and full-length forms of adiponectin mediate specific changes in glucose and fatty acid uptake and metabolism in cardiomyocytes. Cardiovascular Research 75 148-157.
Park SY, Cho YR, Kim HJ, Higashimori T, Danton C, Lee MK, Dey A, Rothermel B, Kim YB, Kalinowski A et al. 2005 Unraveling the temporal pattern of diet-induced insulin resistance in individual organs and cardiac dysfunction in C57BL/6 mice. Diabetes 54 3530-3540.

Peterson LR, Herrero P, Schechtman KB, Racette SB, Waggoner AD, Kisrieva-Ware Z, Dence C, Klein S, Marsala J, Meyer T et al. 2004 Effect of obesity and insulin resistance on myocardial substrate metabolism and efficiency in young women. Circulation 109 2191-2196.

Pineiro R, Iglesias MJ, Gallego R, Raghay K, Eiras S, Rubio J, Dieguez C, Gualillo O, Gonzalez-Juanatey JR \& Lago F 2005 Adiponectin is synthesized and secreted by human and murine cardiomyocytes. FEBS Letters 579 5163-5169.

Purdham DM, Zou MX, Rajapurohitam V \& Karmazyn M 2004 Rat heart is a site of leptin production and action. American Journal of Physiology. Heart and Circulatory Physiology 287 H2877-H2884.

Ramanadham S, Doroudian A \& McNeill JH 1990 Myocardial and metabolic abnormalities in streptozotocin-diabetic Wistar and Wistar-Kyoto rats. Canadian Journal of Cardiology 6 75-82.

Schaffer JE 2003 Lipotoxicity: when tissues overeat. Current Opinion in Lipidology 14 281-287.

Shiomi T, Tsutsui H, Ikeuchi M, Matsusaka H, Hayashidani S, Suematsu N, Wen J, Kubota T \& Takeshita A 2003 Streptozotocin-induced hyperglycemia exacerbates left ventricular remodeling and failure after experimental myocardial infarction. Journal of the American College of Cardiology 42 165-172.

Stanley WC, Recchia FA \& Lopaschuk GD 2005 Myocardial substrate metabolism in the normal and failing heart. Physiological Reviews $\mathbf{8 5}$ 1093-1129.

Vu V, Kim W, Fang X, Liu YT, Xu A \& Sweeney G 2007 Coculture with primary visceral rat adipocytes from control but not streptozotocin-induced diabetic animals increases glucose uptake in rat skeletal muscle cells: role of adiponectin. Endocrinology 148 4411-4419.

Wang P, Lloyd SG, Zeng H, Bonen A \& Chatham JC 2005 Impact of altered substrate utilization on cardiac function in isolated hearts from Zucker diabetic fatty rats. American Journal of Physiology. Heart and Circulatory Physiology 288 H2102-H2110.

\section{Received in final form 8 September 2008 Accepted 10 September 2008 Made available online as an Accepted Preprint 11 September 2008}

